\title{
Optimization of Differential Diagnosis of Inflammatory Bowel Diseases Based on an Integrated Assessment of Oral Mucosa Status
}

\author{
DOI 10.17691/stm2015.7.1.11
}

Received December 1, 2014

N.S. Robakidze, PhD, Associate Professor, Acting Head of the Department of Orthopedic Dentistry':

Senior Research Fellow, Research Institute of Biomedical Technologies

II.I. Mechnikov North-Western State Medical University, 41 Kirochnaya St., Saint Petersburg, 191015:

Russian Federation;

${ }^{2}$ Nizhny Novgorod State Medical Academy, 10/1 Minin and Pozharsky Square, Nizhny Novgorod, 603005, Russian Federation

The objective of the investigation was to improve the differential diagnosis of inflammatory bowel diseases, on the basis of an integrated assessment of morphological data, the findings of optical imaging of tissues, and immunohistochemical examinations of the oral mucosa.

Materials and Methods. We studied morphological and immunohistochemical characteristics of the oral mucosae in each of 26 patients with Crohn's disease (CD) and 17 patients with ulcerative colitis (UC). We performed both visual comparisons and quantitative assessments of cross-polarization optical coherence tomography (CP OCT) images and morphological data on collagen fibers obtained from polarization microscopy using picrosirius red staining.

Results. We suggest a new and objective differential diagnostic criterion for CD and UC which makes use of the condition of the oral mucosa, based on a quantitative assessment of CP OCT images (by calculation of the integral depolarization factor). In accordance with the results of immunohistochemical studies, we have revealed the most significant indices, and that these can be used as differential diagnostic criteria in CD and UC. We have developed a mathematical model including the four most immunohistochemical significant attributes (relative area of staining, the degree of CD16 expression, CD31 staining intensity and the degree of CD57 expression). We have determined a threshold level for the area of tryptase expression by the mast cells of the buccal mucosa, which achieves the maximum differential diagnostic accuracy for the CD and UC indices. The most significant characteristics of the immunohistochemical method exceed the diagnostic efficiency parameters of CP OCT.

Conclusion. Given that CP OCT is non-invasive and has high diagnostic accuracy, we recommend using a quantitative assessment of CP OCT images as the first stage of an operational differential diagnosis of the diseases, based on the condition of the oral mucosa. In the case of inconclusive results, an oral mucosa biopsy, followed by morphological and immunohistochemical examination, would be expedient.

Key words: Crohn's disease; ulcerative colitis; oral mucosa; optical coherence tomography; immunohistochemical examination.

Introduction. Crohn's disease (CD) and ulcerative colitis (UC) are two types of inflammatory bowel disease (IBD). By number of clinical presentations CD and UC are, to some extent, similar, however, each has a variety of specific features. While both diseases are characterised by nonspecific immune inflammation, UC affects only the large intestine (except in retrograde ileitis), the inflammation process always involves the rectum, and at most, the inflammation is diffuse and limited to the mucosa (except in fulminant colitis). CD is characterised by transmural segmental inflammation of any part of the gastrointestinal tract. The diagnostics of Crohn's disease and ulcerative colitis are based on a combination of clinical, endoscopic, histological and radiological data [1, 2]. Differential diagnosis of these diseases can be troublesome, when in $C D$ the inflammation is confined to the large intestine, and the endoscopic and histological signs of CD and UC are therefore almost identical, since there is no socalled gold standard. Unfortunately, not all CD patients have such pathognomonic symptoms, as "cobble-stone pavement", punched-out and aphthoid ulcers, strictures or fistulas, which would help to verify the diagnosis. CD may proceed for years under a mask of UC or not classified colitis, and frequently, the disease becomes obvious only if complications develop [3].

Both diseases are progressive: $C D$ results in the development of strictures and penetrating, including perianal, complications, with a risk of surgery being required; progressive UC is associated with the extended involvement of the large intestine, and the risk of dysplasia and colorectal carcinoma. The necessity for a precise diagnosis is dictated by the similarity in the natural course of each disease, and also by their different responses to drug therapy, especially in relation to the choice of medications and the likely extent of surgery required. Microscopic analysis of numerous colorectal biopsies enables a reliable CD diagnosis in only $64 \%$ of cases, while for UC the corresponding figure is $74 \%$. If the disease is limited to the large intestine, a definitive

Corresponding author: Robakidze Natalia S., e-mail: rona24@list.ru 
diagnosis of $U C$ or $C D$ cannot be made in $5-10 \%$ of cases, even in specialist clinics [4]. In such a situation, the European Crohn's and Colitis Organization (ECCO) advises the use of the term "not classified colitis", when UC and CD cannot be differentiated after studying a case history, the analysis of clinical presentations, the histological examination of numerous biopsies of the intestinal mucosa or a radiological study. Attempts to differentiate UC and CD using pANCA and ASCA antibodies have not been successful, since the sensitivity of the technique is only $40-60 \%$, which reduces its value in non-classified colitis $[5,6]$.

According to most researchers, UC and CD differ in the types of cellular immune response $[7,8]$. Immunohistochemical examinations explain some problems of the pathogenesis of both diseases; however, their position in a diagnostic algorithm is still unclear [9].

There are different opinions on the involvement of the oral mucosa $(\mathrm{OM})$ in the inflammatory process in CD. Some authors consider oral diseases in relation to $\mathrm{CD}$-specific localisation, while others see them only as extra-intestinal manifestations [10, 11]. Previous studies carried out on resected intestinal tissues, and later - in vivo endoscopic materials, have shown the diagnostic capabilities of optical coherence tomography (OCT) in the differential diagnosis of $C D$ and UC [12]. Moreover, these authors studied the correlation of OCT findings with the results of histological studies, determined the criteria for OCT images in different types of IBD (transmural inflammation with destruction of the structural layering of the intestinal wall in $C D$, and surface inflammation with clearly differentiated layers in UC), and demonstrated the high diagnostic accuracy of OCT. The use of such OCT modification as cross-polarization OCT (CP OCT) has made it possible, both to carry out in vivo study of microstructure tissue changes, and also to obtain an overview of changes in collagen structure, based on an assessment of its polarization characteristics.

The value of OCT diagnostic information has been demonstrated in many studies in the early diagnosis of dental and soft oral tissue diseases [13-15]. The search for new differential diagnostic criteria in the oral cavity as manifestations of IBD, using modern research techniques, is relevant in extending the concept of the pathogenesis of combined oral and intestinal pathology, and in developing novel approaches to the differential diagnosis of $C D$ and UC.

The objective of the investigation was to improve the differential diagnostics of inflammatory bowel diseases, based on an integrated assessment of morphological data, the findings of in vivo optical imaging of tissues, and immunohistochemical examinations of the oral mucosa.

Materials and Methods. With the aim of studying the morphological and immunohistochemical characteristics of the OM, we examined 26 patients with $\mathrm{CD}$ and 17 patients with UC, who were being treated in the City IBD Diagnostic and Medical Centre, Saint Petersburg, from 2008 to 2013. The control group consisted of 20 subjects with neither IBD nor OM diseases.

The study complies with the declaration of Helsinki and approved by the Ethics Committee of I.I. Mechnikov NorthWestern State Medical University. Written informed consent was obtained from all patients.

As a preliminary, all patients underwent ileocolonoscopy with multiple biopsy, followed by a histological and immunohistochemical study of the bioptates of the large and small intestinal mucosae. For the histological examination of the OM we chose the buccal area as an example of a tissue covered by stratified squamous epithelium and having a marked connective tissue subepithelial layer. Bioptates were taken from a point situated $0.5 \mathrm{~cm}$ above the third molar occlusal surface. The material obtained was placed on filter paper and fixed in 10\% neutral buffered formaldehyde solution. The subsequent paraffin embedding was performed according to standard practice. A series of sections $5 \mu \mathrm{m}$ in thickness was obtained in a single-stage procedure. The sections were stained with hematoxylin and eosin, as well as with van Gieson's picrifuchsin stain. Using survey microscopy we assessed the stromal epithelial relationships, as well as the intensity and character of any inflammatory response.

We obtained 166 CP OCT images of buccal mucosae from $16 \mathrm{CD}$ patients and 10 UC patients using an OKT1300-U (BioMedTech, Russia) with an operating wavelength of $1300 \mathrm{~nm}$. Performance characteristics are tissue incident power of $3 \mathrm{~mW}$, depth resolution of $15-20 \mu \mathrm{m}$, lateral resolution of $25 \mu \mathrm{m}$, scanning depth of $\sim 1.5 \mathrm{~mm}$, frame rate for B-scan image pair of $200 \times 256$ pixels each is 0.5 frames per second. More detailed system descriptions, including the optical phase retardation particulars to enable $\mathrm{CP}$ operation, have been published previously $[16,17]$. CP OCT combines two images containing different information related to the internal structure of the tissues. The image obtained with the initial polarization provides data on the scattering by tissue components, and is primarily analyzed using such criteria as the presence/absence of layering. OCT signal in the orthogonal image indicates the presence/absence of anisotropic tissue components such as collagen fibers (CF). Each region of interest was scanned several times until a repeatable image was obtained; then a biopsy was taken from this area for morphological analysis. We visually compared and quantitatively assessed the $\mathrm{CP}$ OCT images and the morphological data of the CF using polarization microscopy of the picrosirius red stained histological specimens. The quantitative assessment of the CP OCT images consisted of the automatic calculation of the integral depolarisation factor (IDF) - a ratio of the OCT signal in orthogonal image to the analogous value calculated in the initial polarization image [18]. The quantitative processing of the digital images consisted of an assessment of the average intensity of 72 histological specimens of buccal mucosae stained with picrosirius red and photographed in polarized light under similar conditions. Evaluation of the intensity and thickness of the CF in the images was carried out according to the technique described by Giattina et al. [19], using Photoshop 7.0.1.

Immunohistochemically, the OM and intestinal bioptates were studied using monoclonal murine antibodies (Dako 
Company, Denmark) to CD5 (to reveal the T-lymphocytes), CD20 (to reveal B-lymphocytes); CD16 and CD57 (to reveal NK-cells), CD31 (to reveal vascular endothelium), and tryptase (to reveal mast cells). The findings were quantitatively assessed using a system of computerassisted assay of the microscopic images, consisting of a Nikon Eclipse E400 microscope (Japan), a Nikon DXM1200 digital camera (Japan), and a personal computer with ACT-1, version 2.12 and Videotest-Morphology 5.2 software (Russia). The marker content was estimated over three fields of vision at $\times 200$ magnification to obtain an average staining intensity (over a range from 0 to 255 relative units $(\mathrm{RU})$; negative expression being characterised by a value of 255) and an index of the relative area of immunohistochemical staining. The expression of the relative area (S, \%) was calculated as the ratio between the area of the immunopositive cells to the total area of the visual field, and expressed in percentage terms.

Results and Discussion. Based on the morphological and immunohistochemical analyses we studied, the pattern of inflammatory response in the two types of IBD determined the frequency of the individual attributes, and revealed the differences between the groups. On the basis of the above data we established differential diagnostic criteria and assessed their operational characteristics.

Histological studies showed that, among other morphological OM characteristics in IBD, mononuclear (primarily, lymphoid) infiltration was determined more frequently: in $94.4 \%$ of cases in CD, and in $66.7 \%$ in UC $\left(\chi^{2}=6.44 ; p=0.01\right)$. Acanthosis and epithelial dystrophy occurred less frequently in both groups $(p>0.10)$. In addition, focality of infiltrate was a highly specific sign for CD. Focal infiltrate was revealed in $94.4 \%$ of CD cases, but in only $33.3 \%$ in UC. One third of UC patients had scanty diffuse lymphoid infiltration. Mononuclear infiltration of tissues outside the bowel suggests an autoimmune character of the IBD [20], and to be more marked in CD [21].
The capabilities of CP OCT enable us to assess microstructural OM changes in the IBD. CP OCT images of $C D$ patients show morphological signs of mucosal inflammation, verified histologically in $100 \%$ of the bioptates. Compared to the norm, the CP OCT images in initial polarization image demonstrate either contrast attenuation, or no contrast, for the epithelium and lamina propria, and no clear layering. CP OCT images of UC patients were characterized by a slight contrast decrease of the clearly differentiated optical layers of the epithelium and lamina propria, and maintained the layered organization of the mucosa (Figure 1).

Given the tendency of the gastrointestinal mucosa to show fibrosis in CD [22], we estimated the microstructural changes of the OM in patients with $\mathrm{CD}$ and UC. OCT orthogonal polarization images from $C D$ patients, when compared to the norm, and to UC patients, showed an increased signal intensity from fibrosing collagen in the lamina propria, comparable to the increased intensity of brightness of the collagen fibers and bundles in polarized light on the histological picrosirius red stained specimens, indicating excessive collagen accumulation in the connective tissue stroma.

To improve the diagnostic accuracy of the technique, in addition to the visual assessment of the pathological processes, we carried out a quantitative analysis of the images, by calculating the IDF. We revealed an increased OCT signal from fibrosing collagen of the lamina propria in CD patients compared to the OCT signal in UC patients. We found that the IDF in CD differs significantly from that in UC $(0.17 \pm 0.01$ versus $0.13 \pm 0.01 ; p<0.05)$.

Significant differences in the quantitative index of the depolarization of the probing radiation indicate different structural and spatial organization changes in the stromal CF of the mucosae for the different diseases, with excessive CF accumulation and thickening (marked fibrosis) in CD, but only slight fibrosis in UC. Thus, the IDF can be used to
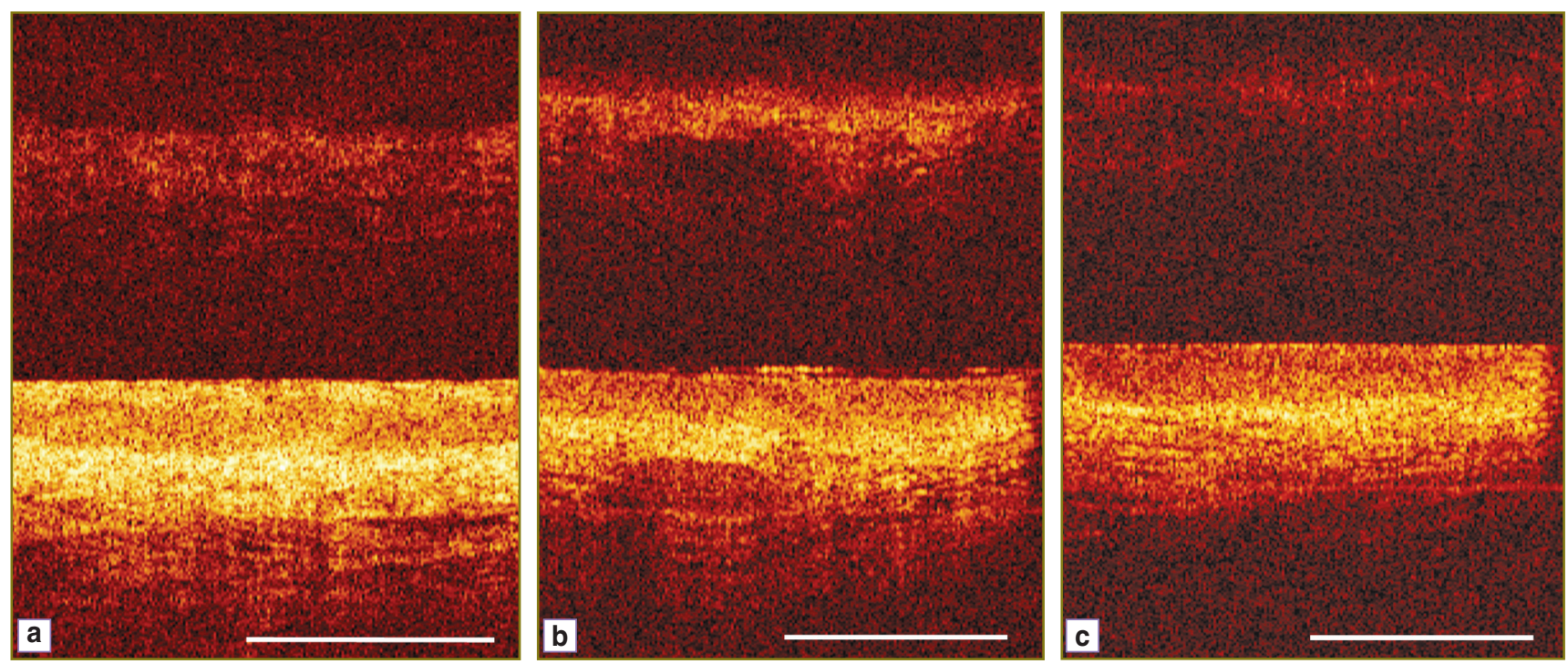

Figure 1. CP OCT images (upper panel — orthogonal polarization, lower panel — initial polarization) in normal tissues (a), in Crohn's disease (b), in ulcerative colitis (c). Bar $1 \mathrm{~mm}$ 
diagnose degrees of fibrosis, and this is consistent with the findings of the morphometric study showing the difference in CF thickness and density in the two diseases. There is a high correlation coefficient between the IDF and the mean intensity of the histological picrosirius red stained specimens $(r=0.72 ; p=0.0001 ; n=26)$.

We calculated the indices of diagnostic efficiency for various IDF values in increments of 0.01 . An IDF equal to 0.13 was found to provide the best index of diagnostic efficiency in the differential diagnosis of $C D$ and $U C$. A definitive diagnostic rule for differential diagnosis was formulated: if the IDF is $\geqslant 0.13$, then a patient has CD; if the IDF is $<0.13-\mathrm{UC}$. The sensitivity of the CP OCT differential diagnostic technique for $C D$ is $88.9 \%$, the specificity is $70.6 \%$, the predictive value of a negative test is $61.5 \%$, and the predictive value of a positive test is $92.3 \%$. The overall diagnostic accuracy is $76.9 \%$.
In the control patients immunohistochemical study of buccal mucosa bioptates showed no expression of CD5, CD16, or CD20 and only low expression of CD31 and CD57. On the other hand, all the cell types under study were revealed, with different frequencies, in both groups with IBD (Figures 2-4). We found significant differences in CD and UC for the degree of expression of CD16, CD31 and CD57. The extent of expression of each of these markers in the oral cavity was higher in CD than in UC, which indicates the complete activation of the cell element of the immune system and moderate inflammation activity in the oral cavity in the case of CD (Table 1).

For the purpose of differential diagnosis we used discriminate function analysis, which enabled us to identify the most significant indices for use as differential diagnostic criteria of CD and UC. The indices were included in a discriminant equation based on Fisher's F-ratio test. The
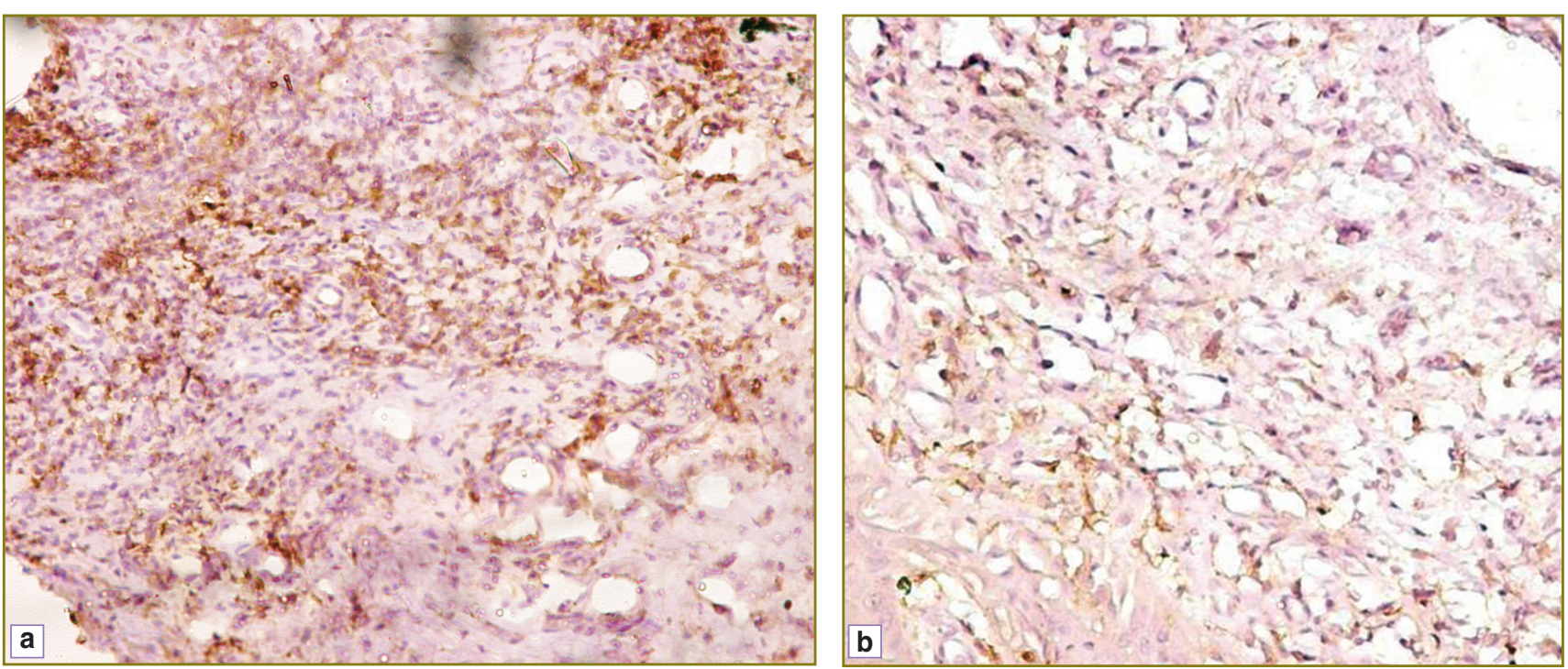

Figure 2. Expression of CD16 in buccal mucosa bioptate: in Crohn's disease (a); in ulcerative colitis (b); $\times 200$


Figure 3. Expression of CD31 in buccal mucosa bioptate: in Crohn's disease (a); in ulcerative colitis (b); $\times 200$ 

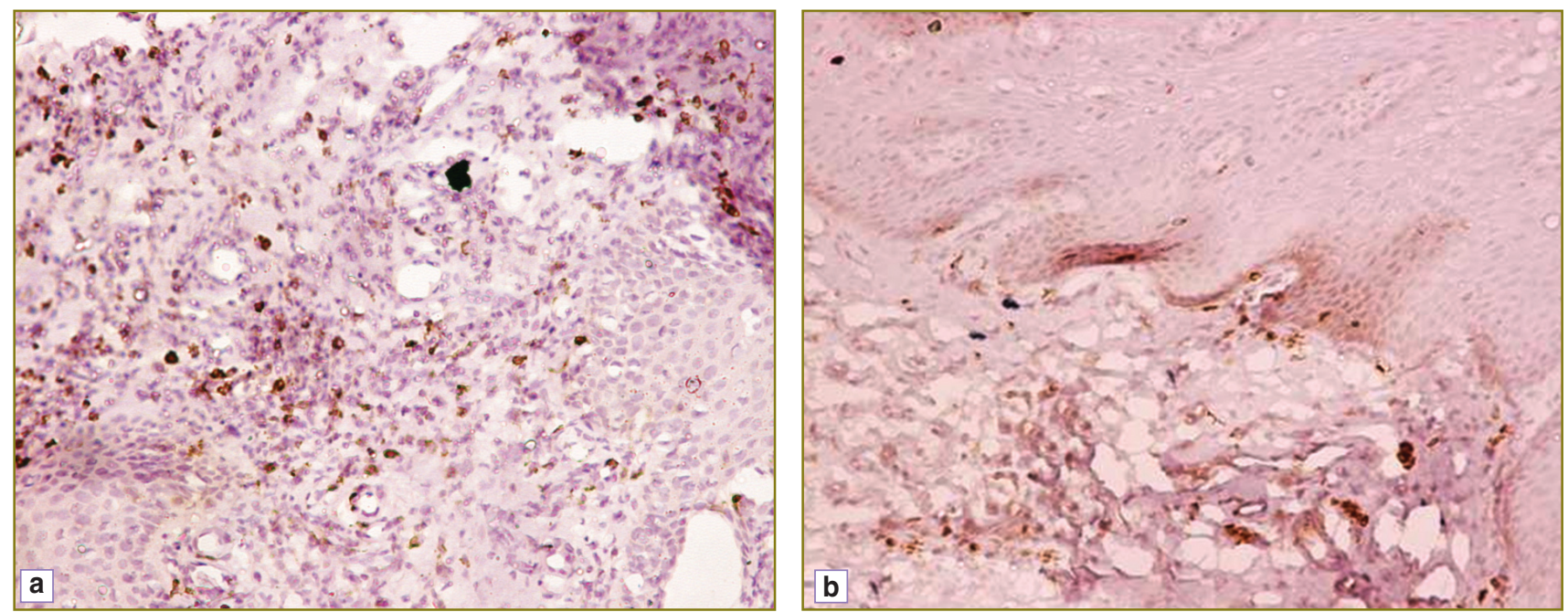

Figure 4. Expression of CD57 in buccal mucosa bioptate: in Crohn's disease (a); in ulcerative colitis (b); $\times 200$

Table 1

Expression indices of immunohistochemical markers in the buccal mucosa

\begin{tabular}{clccc}
\hline & \multicolumn{1}{c}{ Parameter } & Crohn's disease $(\mathbf{n = 2 6 )}$ & Ulcerative colitis ( $\mathbf{n = 1 7 )}$ & $\mathbf{p}$ \\
\multirow{2}{*}{ CD5 } & Relative area (S, \%) & $1.11 \pm 0.34$ & $1.61 \pm 0.51$ & $>0.10$ \\
\cline { 2 - 5 } & Average staining intensity (RU) & $226.69 \pm 8.94$ & $191.81 \pm 15.49$ & 0.04 \\
\multirow{2}{*}{ CD16 } & Relative area (S, \%) & $4.61 \pm 0.93$ & $3.07 \pm 0.66$ & $>0.10$ \\
\cline { 2 - 5 } & Average staining intensity (RU) & $180.63 \pm 11.32$ & $229.06 \pm 6.92$ & 0.003 \\
\hline \multirow{2}{*}{ CD20 } & Relative area (S, \%) & $0.24 \pm 0.09$ & $0.71 \pm 0.23$ & 0.03 \\
\cline { 2 - 5 } & Average staining intensity (RU) & $242.51 \pm 6.06$ & $229.39 \pm 8.00$ & $>0.10$ \\
\hline \multirow{2}{*}{ CD31 } & Relative area (S, \%) & $2.36 \pm 0.30$ & $1.46 \pm 0.19$ & 0.03 \\
\cline { 2 - 5 } & Average staining intensity (RU) & $140.57 \pm 8.57$ & $198.38 \pm 12.13$ & 0.001 \\
\hline \multirow{2}{*}{ CD57 } & Relative area (S, \%) & $2.00 \pm 0.66$ & $0.26 \pm 0.09$ & 0.04 \\
\cline { 2 - 4 } & Average staining intensity (RU) & $153.69 \pm 10.38$ & $231.12 \pm 6.39$ & $<0.001$ \\
\hline
\end{tabular}

analysis carried out resulted in a mathematical model including the four most significant features:

\section{$D F=2.036-0.491 \cdot z I C D 57+0.00274 \cdot I N T C D 31-0.418 x$ $\times z I C D 16+0.297 \cdot z S C D 16\left(\mathrm{R}^{2}=0.72 ; \mathrm{F}=27.98 ; \mathrm{p}<0.0001\right)$,}

where $D F$ is the discriminant function (non-dimensional value); zICD57 is the degree of expression (code: 1 - low, 2 - moderate, 3 - high); INTCD31 is the average staining intensity (absolute unit); $z I C D 16$ is the degree of expression (code: 1 - low, 2 - moderate, 3 - high); $z S C D 16$ is the presence of staining (code: 0 - there is no stained area, 1 - there is a stained area).

According to the technique we have developed, the IBD diagnosis procedure consists of the calculation of the discriminant function for each patient, with a subsequent comparison of the result with a threshold value. The maximum indices of diagnostic efficiency were found to be achieved if the value was 1.46. To make a diagnosis, we formulated the decision diagnostic rule: if the discriminant function index is $\leqslant 1.46$, the most probable diagnosis is $C D$; and if the index is $>1.46$, the presence of $U C$ is indicated. We checked the model by comparing the actual and predicted diagnoses of CD and UC. The technique sensitivity is $100 \%$, its specificity - $92.3 \%$, the predictive value of a negative test $-100 \%$, the predictive value of a positive test $-89.5 \%$. The overall diagnostic accuracy is 95.3\%.

Given the participation of mast cells in the inflammatory responses [23], in order to search for additional differential diagnostic criteria of $C D$ and UC we carried out an immunohistochemical study of the mast cells of the buccal mucosa, using antibodies to tryptase. Mast cells are known to be involved in the remodeling of tissues in CD. Chemokines released by mast cells can selectively attract macrophages and $\mathrm{CD}^{+}$cells, worsening any inflammatory response in the damage area [24].

We revealed significant differences in the area and intensity of tryptase expression in CD and UC (Table 2). The area of tryptase expression proved to be the key index 
Table 2

Indices of tryptase expression by mast cells of the buccal mucosa

\begin{tabular}{|lcccc}
\hline \multicolumn{1}{c}{ Parameter } & Crohn's disease $(\mathbf{n}=\mathbf{1 2})$ & Ulcerative colitis (n=8) & $\mathbf{t}$ & $\mathbf{p}$ \\
\hline Relative area (S, \%) & $16.60 \pm 1.74$ & $4.96 \pm 2.01$ & 4.31 & $<0.001$ \\
\hline Average staining intensity (RU) & $174.01 \pm 1.39$ & $159.83 \pm 1.49$ & 3.59 & 0.002 \\
\hline
\end{tabular}

$(F=25.18 ; p<0.0001)$, and this can be used as a diagnostic criterion to differentiate between $C D$ and UC. When calculating the diagnostic efficiency indices for different values of the area of tryptase expression we found that the maximum efficiency of CD and UC differential diagnosis was achieved when the index was $7.18 \%$. To make a diagnosis, we formulated the decision diagnostic rule: if the expression index is $\geqslant 7.18 \%$, then a patient has CD; if $<7.18 \%$, it is concluded that the patient has UC. The technique sensitivity is $100 \%$, its specificity - $92.3 \%$, the predictive value of a negative test $-100 \%$, and the predictive value of a positive test $-87.5 \%$. The overall prediction accuracy is $95.0 \%$. The operational characteristics of the immunohistochemical method indicate the high accuracy of the technique in relation to $C D$ diagnosis, and exceed the performance of CP OCT in diagnostic efficiency.

Taking into consideration the effect of mast cells on collagen metabolism in reparative fibrosis [25] we carried out an analysis of the relationship between the IDF and the area of tryptase expression, and revealed a correlation between the IDF indicating CF accumulation in fibrosis, and the area of tryptase expression $(r=0.53 ; p=0.019 ; r s=0.52$; $\mathrm{p}=0.022 ; \mathrm{n}=20$ ).

Conclusion. Based on the assessment of morphological data, the findings of optical coherence tomography and immunohistochemistry, we have distinguished differential diagnostic criteria for Crohn's disease and ulcerative colitis using the state of the oral cavity. The criteria enable us to optimize the algorithm for the differential diagnosis of inflammatory bowel disease. When carrying out an integrated diagnostic examination of a patient one should follow certain diagnostic procedures. Taking into consideration the non-invasiveness and particularly high diagnostic accuracy of CP OCT we recommend using a quantitative assessment of CP OCT images (calculation of the integral depolarization factor) as the first stage of operational differential diagnosis on the basis of the condition of the oral mucosa. In the case of inconclusive results, an oral mucosa biopsy, followed by morphological and immunohistochemical studies would be expedient.

Study Finding. This study was supported the Russian Scientific Fund (Agreement No.14-15-00538).

Acknowledgements. The author thanks the BioMedTech company for the use of the OKT1300-U device in the study; and Drs. O.B. Stchukina (I.I. Mechnikov North-Western State Medical University, Saint Petersburg), I.M. Kvetny (D.O. Ott Research Institute of Obstetrics and Gynecology, Saint Petersburg), N.D. Gladkova, E.B. Kiseleva (Nizhny Novgorod State Medical Academy, Nizhny
Novgorod), and M.Y. Kirillin (Institute of Applied Physics of the Russian Academy of Sciences, Nizhny Novgorod) for their advice.

Conflict of Interest. The authors declare no conflicts of interest related to this study.

\section{References}

1. Assche G., Dignass A., Reinisch W., van der Woude C.J., Sturm A., De Vos M., et al. The second European evidence-based consensus on the diagnosis and management of Crohn's disease: special situations. $J$ Crohn's Colitis 2010; 41(1): 63-101, http://dx.doi.org/10.1016/ j.crohns.2009.09.009.

2. Dignass A., Lindsay J.O., Sturm A., Windsor A., Colombel J.F., Allez M., et al. Second European evidencebased consensus on the diagnosis and management of ulcerative colitis. Part 2: current management. J. Crohn's Colitis 2012; 6(10): 991-1030, http://dx.doi.org/10.1016/ j.crohns.2012.09.002.

3. Stchukina O.B. Indeterminate colitis: current state of the problem. Consilium Medicum 2008; 10(8): 77-79.

4. Bentley E., Jenkins D., Campbell F., Warren B. How could pathologists improve the initial diagnosis of colitis? Evidence from an international workshop. J Clin Pathol 2002; 55(12): 955-960, http://dx.doi.org/10.1136/jcp.55.12.955.

5. Joossens S., Reinisch W., Vermeire S., Sendid B., Poulain D., Peeters M., Geboes K., Bossuyt X., Vandewalle P., Oberhuber G., Vogelsang H., Rutgeerts P., Colombel J.F. The value of serologic markers in indeterminate colitis: a prospective follow-up study. Gastroenterology 2002; 122(5): 1242-1247, http://dx.doi.org/10.1053/gast.2002.32980.

6. Mow W.S., Landers C.J., Steinhart A.H., Feagan B.G., Croitoru K., Seidman E., Greenberg G.R., Targan S.R. Highlevel serum antibodies to bacterial antigens are associated with antibiotic-induced clinical remission in Crohn's disease: a pilot study. Dig Dis Sci 2004; 491(7-8): 1280-1286, http://dx.doi. org/10.1023/b:ddas.0000037824.66186.e2.

7. Belousova E.A. Yazvennyy kolit i bolezn' Krona [Ulcerative colitis and Crohn's disease]. Tver: OOO Izdatel'stvo "Triada"; 2002.

8. Brown S.J., Mayer L. The immune response in inflammatory bowel disease. Am J Gastroenterol 2007; 102(9): $\quad 2058-2069, \quad$ http://dx.doi.org/10.1111/j.15720241.2007.01343.x.

9. Strioga M., Pasukoniene V., Characiejus D. CD8 ${ }^{+}$ $\mathrm{CD}^{-} 8^{-}$and $\mathrm{CD}^{+} \mathrm{CD}^{+} 7^{+} \mathrm{T}$ cells and their role in health and disease. Immunology 2011; 134(1): 17-32, http://dx.doi. org/10.1111/j.1365-2567.2011.03470.x.

10. Geboes K. Crohn's disease, ulcerative colitis or indeterminate colitis - how important is it to differentiate? Acta Gastroenterol Belg 2001; 64(2): 197-200. 
11. Lankarani K.B., Sivandzadeh G.R., Hassanpour S. Oral manifestation in inflammatory bowel disease: a review. World $J$ Gastroenterol 2013; 19(46): 8571-8579.

12. Shen B., Zuccaro G., Gamilich T.L., Gladkova N., Lashner B.A., Delaney C.P., Connor J.T., Remzi F.H., Kareta M., Bevins C.L., Feldchtein F., Strong S.A., Bambrick M.L., Trolli P., Fazio V.W. Ex vivo histologycorrelated optical coherence tomography in the detection of transmural inflammation in Crohn's disease. Clin Gastroenterol Hepatol 2004; 2(9): 754-760, http://dx.doi.org/10.1016/S15423565(04)00346-5.

13. Gladkova N., Kiseleva E., Robakidze N., Balalaeva I., Karabut M., Gubarkova E., Feldchtein F. Evaluation of oral mucosa collagen condition with cross-polarization optical coherence tomography. J Biophotonics 2013; 6(4): 321-329, http://dx.doi.org/10.1002/jbio.201200059.

14. Ganganna K., Shetty P., Shroff S.E. Collagen in histologic stages of oral submucous fibrosis: a polarizing microscopic study. J Oral Maxillofac Pathol 2012; 16(2): 162166, http://dx.doi.org/10.4103/0973-029X.98446.

15. Lee C.K., Tsai M.T., Lee H.C., Chen H.M., Chiang C.P., Wang Y.M., Yang C.C. Diagnosis of oral submucous fibrosis with optical coherence tomography. J Biomed Opt 2009; 14(5): 054008, http://dx.doi.org/10.1117/1.3233653.

16. Feldchtein F.I., Gelikonov V.M., Gelikonov G.V. Polarization-sensitive common path optical coherence reflectometry/tomography device. Patent US 7,728,985 B2. 2010.

17. Gelikonov V.M., Gelikonov G.V. New approach to crosspolarized optical coherence tomography based on orthogonal arbitrarily polarized modes. Laser Phys Lett 2006; 3(9): 445451, http://dx.doi.org/10.1002/lapl.200610030.

18. Gubarkova E.V., Kirillin M.Y., Sergeeva E.A.,
Kiseleva E.B., Snopova L.B., Prodanets N.N., Sharabrin E.G., Shakhov E.B., Nemirova S.V., Gladkova N.D. Cross-polarization optical coherence tomography in evaluation of atherosclerotic plaque structure. Sovremennye Tehnologii v Medicine 2013; 5(4): 45-55.

19. Giattina S.D., Courtney B.K., Herz P.R., Harman M., Shortkroff S., Stamper D.L., Liu B., Fujimoto J.G., Brezinski M.E. Assesment of coronary plaque collagen with polarization sensitive optical coherence tomography (PS-OCT). Int J Cardiol 2006; 107(3): 400-409, http://dx.doi.org/10.1016/ j.ijcard.2005.11.036.

20. Vorob'ev G.I., Khalif I.L. Nespetsificheskie vospalitel'nye zabolevaniya kishechnika [Non-specific inflammatory bowel disease]. Moscow: Miklosh; 2008; 400 p.

21. Veloso T.F. Extraintestinal manifestations of inflammatory bowel disease: do they influence treatment and outcome? World J Gastroenterol 2011; 17(22): 2702-2707.

22. Graham M.F., Diegelmann R.F., Elson C.O., Lindblad W.J., Gotschalk N., Gay S., Gay R. Collagen content and types in the intestinal strictures of Crohn's disease. Gastroenterology 1988; 94(2): 257-265.

23. Walsh L.J. Mast cell and oral inflammation. Crit Rev Oral Biol Med 2003; 14(3): 188-198, http://dx.doi.org/10.1177/ 154411130301400304.

24. Middel P., Thelen P., Blaschke S., Polzien F., Reich K., Blaschke V., Wrede A., Hummel K.M., Gunawan B., Radzun H.J. Expression of the T-cell chemoattractant chemokine lymphotactin in Crohn's disease. Am J Pathol 2001; 159(5): 1751-1761, http://dx.doi.org/10.1016/S00029440(10)63022-2.

25. Pujari R., Vidya N. Mast cell density in oral submucous fibrosis: a possible role in pathogenesis. Int $\mathrm{J}$ Health $\mathrm{Sci}$ (Qassim) 2013; 7(1): 23-29. 